\title{
Focus on Cell cycle and DNA damage
}

\section{How cells accurately duplicate and segregate their genetic information remains a topic of intense research. A series of specially commissioned articles in this issue presents recent insights into different aspects of the cell division cycle and genomic surveillance.}

DNA replication, mitotic spindle formation, chromosome segregation and cytokinesis must be carefully controlled to ensure that all genetic information is passed over to the next cell generation. Although the core cell cycle machinery has been worked out, and the cyclin-dependent kinases and cyclins entered the textbooks a long time ago, we still lack a complete understanding of how events of the cell cycle are executed and coordinated. In mitosis, the microtubule-based spindle organizes duplicated chromosomes to allow the segregation of two identical sets to daughter cells. In most cells, centrosomes, each consisting of two centrioles, are the main organizers of microtubules, influencing spindle assembly and chromosome segregation. Centrosomes, like chromosomes, need to be replicated only once per cell cycle and segregated when the cell divides. Erich Nigg and Tim Stearns discuss recent insights into the centrosome life cycle, the potential role of centrosomes in genomic stability and aspects of asymmetry in centriole architecture and segregation. Before separation occurs in anaphase, however, duplicated sister chromatids are held tightly together. The 'DNA glue' is provided by the cohesin complex, vital also for cohesion during DNA repair and replication. Mechanistically, cohesin has been suggested to form a ring that entraps the DNA, but the precise structure of the ring, and how it would come on and off DNA remains a mystery. Kim Nasmyth describes findings on cohesin function and proposes a model to explain cohesin loading onto - and dissociation from - DNA.

Another aspect of cell cycle progression is the existence of safeguarding mechanisms called 'checkpoints' that ensure everything is in order before allowing the next event to proceed. The spindle checkpoint (or spindle assembly checkpoint, SAC) monitors the alignment of the full set of chromosomes on the mitotic spindle before anaphase can take place. In the twenty years since the isolation of the first spindle checkpoint genes in yeast, much has been learned about the molecular players in the SAC. In a Historical Perspective, Andrew Murray discusses these advances and outlines the most important questions that remain to be resolved.

Repairing damaged DNA is crucial for genomic stability. Recent research has revealed that chromatin undergoes dramatic changes in response to DNA damage. This causes a massive accumulation of proteins in 'nuclear foci. Jiri Lukas, Claudia Lukas and Jiri Bartek review the numerous post-translational modifications of chromatin proteins following DNA damage, and their potential biological function.

In addition to these articles, an accompanying online library on this topic presents selected research papers and reviews from Nature journals. We thank our authors and reviewers for their contributions and hope that our readers will share our enthusiasm for this Focus issue.

\section{UK Parliament comments on peer review}

\section{Recognizing the importance of sound scientific advice to the government, the UK Parliament has examined the peer review system.}

In July, the House of Commons Science and Technology Committee published a comprehensive report "Peer review in scientific publication" based on input from researchers, funding bodies and publishers. Parliament's undertaking to understand and assess the value of the peer review process, which remains fundamental to ensuring quality in life science publications, should be applauded. The committee concludes that despite its flaws, pre-publication peer review is vital and cannot be dismantled. However, the report also highlights much-needed improvements to the process including training of early career scientists in peer review. The committee recognizes the crucial role of reviewers and their tremendous efforts and calls for better recognition of this work, but does not make any concrete proposals to this end. As we have previously discussed in these pages ("Reviewing refereeing"), we agree that this fundamental contribution should be appropriately acknowledged, not just by journal editors and publishers, but also by tenure-granting committees, funding agencies and other bodies that evaluate researcher performance and their contributions to a field.

The report also discusses avenues for reducing the burden on reviewers, such as editorial pre-screening, efforts to increase a journal's 'reviewer pool', and the possibility to transfer manuscripts between journals together with the referee reports. These approaches are indeed employed by Nature journals to facilitate a constructive and efficient peer review process for authors and referees alike. The committee rightly notes the important role of post-publication review through online commentary and of social media tools in communicating published work and discussing its merits and weaknesses. To this end, we have recently begun highlighting Faculty of 1000 coverage of our papers on our homepage. Of course, no analysis of peer review is complete without a discussion of impact factors or the pressures on researchers to publish in high-impact journals. The committee warns against using impact factors as a proxy for measuring the quality of a publication, and exhorts funders and research institutions to assess individual works. We agree; impact factors of journals are an imperfect proxy for measuring the significance of a study, and there is no substitute for evaluating an individual publication on its own merit. Finally, the report discusses research integrity. Journals, including this one, have some means to detect data manipulation (see "Guide to Authors: calling all authors!" and "Combating scientific misconduct"). However, the committee finds the oversight of research integrity at other levels unsatisfactory. Their call for the establishment of an oversight body in the UK, and their recommendation that research institutes have a formal process to deal with ethical issues should be implemented.

In conclusion, the pre-publication peer review system, in spite of its deficiencies, is likely to remain an integral part of the dissemination of research findings. Not only does the scientific community rely on it, but scientific advice to governments and information to the public should be based on robust data. 